\title{
Sarilumab: patient-reported outcomes in rheumatoid arthritis
}

\section{Chiara Crotti ${ }^{1,2}$ \\ Martina Biggioggero ${ }^{3}$ \\ Andrea Becciolini ${ }^{3}$ \\ Ennio Giulio Favalli ${ }^{3}$}

'Department of Clinical Sciences and Health Community, University of Milan, ${ }^{2}$ Division of Rheumatology, Gaetano Pini Institute, Milan, Italy; ${ }^{3}$ Department of Rheumatology, Gaetano Pini Institute, Milan, Italy
Correspondence: Ennio Giulio Favalli Department of Rheumatology, Gaetano Pini Institute, Via Gaetano Pini 9, Milan 20I22, Italy

Tel +39025829642I

Fax +3902582963I5

Email ennio.favalli@gmail.com
This article was published in the following Dove Press journal: Patient Related Outcome Measures

\begin{abstract}
In the last few decades, strategies for the management of rheumatoid arthritis (RA) have been increasingly oriented toward more comprehensive control of the disease, taking into account even RA extra-articular manifestations, comorbidities, and the patient's perception about the disease. The need for improving the shared decision-making process suggested by European League Against Rheumatism recommendations is leading to an increasing interest in the role of patient-reported outcomes (PROs) beside the usual more objective criteria for defining clinical response based on disease-activity composite indices. Measurement of such PROs as pain or fatigue may be significantly influenced by mood disorders often complicating RA, the pathogenesis of which is deeply interconnected with phlogistic processes mediated by proinflammatory cytokines. IL6 is a pleiotropic mediator involved in neuroendocrine and neuropsychological processes, besides its well known effects on immune, cardiovascular, and metabolic systems. Therefore, there is a growing body of evidence about the efficacy of IL6 blockade in PRO improvement in RA patients. Sarilumab is a monoclonal antibody binding both soluble and membrane-bound IL6R $\alpha$, inhibiting the IL6-mediated signaling pathway with favorable efficacy and safety profile. This review analyzes the importance of PROs in strategies for the management of RA and the pathogenic mechanisms linking IL6 with the patient's perception of the disease. Moreover, the main findings from sarilumab randomized controlled trials are summarized in detail, emphasizing the potential role of this IL6 blocker in the holistic treatment of RA.
\end{abstract}

Keywords: rheumatoid arthritis, interleukin-6, sarilumab, patient reported outcome

\section{Introduction}

Rheumatoid arthritis (RA) is a chronic autoimmune disease affecting almost $1 \%$ of the world's population, ${ }^{1}$ characterized by progressive articular disability, systemic inflammation, and high morbidity. ${ }^{2,3}$ RA primarily affects joints, leads to their destruction and loss of function, negatively influencing quality of life and the ability to perform daily activities. ${ }^{4}$ Moreover, beyond articular involvement, RA is a systemic syndrome frequently associated with extra-articular manifestations and comorbidities, which can affect pulmonary, nervous, cardiovascular, and skeletal systems. ${ }^{5}$ From a pathogenic point of view, the immunological pathways involved in the onset of RA synovitis have been demonstrated to be deeply interconnected with the development of extraskeletal involvement. ${ }^{2}$ As an example, several reports have highlighted the complex correlation between inflammation, common RA systemic symptoms (such as pain or fatigue), and mental health disorders. ${ }^{6}$ Similarly, the impairment in functional and mental status has been demonstrated to be a strong predictor of poor clinical response and low likelihood to achieve low disease activity or remission..$^{7-10}$ Moreover, there is growing evidence 
about the impact of certain proinflammatory cytokines on inducing and worsening mental health disturbances and physical function in RA patients. In particular, IL6 seems to play a pleiotropic role in immune, metabolic, neuroendocrine, and neuropsychological processes, and is deeply involved in the development of the complex systemic RA phenotype. ${ }^{1-16}$ Accordingly, IL6 blockade by tocilizumab has been reported to improve RA-disease activity significantly in both randomized controlled trials (RCTs) and observational real-life studies, ${ }^{17,18}$ and other IL6 blockers have been more recently developed. In particular, sarilumab, a fully human $\operatorname{IgG}_{1}$ monoclonal antibody binding both soluble and membranebound IL6R $\alpha,{ }^{19}$ has demonstrated a favorable efficacy and safety profile ${ }^{20-23}$ and was recently approved by the European Medicines Agency and US Food and Drug Administration for the treatment of RA.

Traditionally, clinical evaluation of RA has been focused on physician-generated measures, such as swollen-joint count or laboratory tests, included in the most commonly used disease-activity indices, such as 28-joint Disease Activity Score $\left(\mathrm{DAS}_{28}\right)$ and Simplified Disease Activity Index. However, in the last decade, strategies for managing RA have aimed toward a more comprehensive evaluation of the disease, even from the patient's perspective, in order better to explore perceptive symptoms, functional status, quality of life, mental status, workability, and treatment tolerability, ${ }^{24,25}$ and better to evaluate the frequent discrepancy between physician and patient evaluation of disease activity. ${ }^{26}$ As a consequence, the consideration and the use of patient-reported outcomes (PROs) in the management of RA has increased progressively, and additional information provided by PROs is now included in the core set of measures recommended to assess disease severity, activity, and response to therapy in both RCTs and daily clinical practice. Considering the previously described potential impact of IL6 in worsening the patient's perception of the disease, PROs have been extensively evaluated in RCTs conducted with the available IL6 inhibitors, with very promising results. The aim of this review is to describe in detail the importance of multiple PRO domains in the holistic measuring of RA clinical response and the evidence supporting the impact of IL6 blockade on these indices, with a specific focus on available sarilumab data.

\section{Use of patient reported outcomes in rheumatoid arthritis: Where are we now?}

An ideal RA-outcome measure should encompass objectives related to symptom resolution and others linked to inflammation control and prevention of structural damage. In this field, significant discrepancies between physicians' and patients' perspective of the disease have been demonstrated, especially in the evaluation of some patient-related health domains, such as pain, fatigue, sleep, and well-being, which can be underestimated by physicians. ${ }^{27-29}$ Furthermore, it has been demonstrated that patients not achieving Boolean remission due to missing one subcriterion most frequently miss the patient global assessment $(\mathrm{PGA}) \leq 1$ criterion $(79.8 \%),{ }^{30}$ confirming the impact of patient perception on the application of a treat-to-target approach. Actually, understanding what patients feel, how they are faring, and how arthritis affects daily life and workability is crucial for an overall comprehension of global patient status and for better planning a therapeutic strategy, which should be based on shared decisions between patients and clinicians. ${ }^{31-33}$ Available PROs usually measured in RA include both generic scores and more specific tools created and validated for RA patients. They cover a wide spectrum of measures, dealing with direct (ie, pain or fatigue) or indirect (ie, sleep or mood disorders) outcomes. According to a recent systematic review of the literature comprising 250 papers (113 RCTs) focused on RA, 138 PROs spread across 14 domains of health have been identified. ${ }^{34}$ The most frequently assessed PROs were function $(68.0 \%)$, pain $(40.0 \%)$, PGA $(49.2 \%)$, and health-related quality of life (HRQoL; 18.4\%). Moreover, other domains, such as fatigue assessment (14.4\%), morning stiffness (10\%), psychological status (9.6\%), productivity losses $(6.4 \%)$, sleep disturbances $(2.4 \%)$, coping $(2 \%)$, and leisure $(0.4 \%)$, were additionally evaluated. The Health Assessment Questionnaire Disability Index (HAQ-DI) was the most frequently used score to report physical function and disability (89.4\%). Visual analog scales (VASs) or numeric rating scales were the tools used for assessing PGA in 50\% and pain in $89 \%$ of the studies considered. Generic HRQoL was evaluated in only $18.4 \%$ of the studies (mainly RCTs) using the Short Form Heath Survey (SF36). These results are consistent with a previous similar review, ${ }^{35}$ confirming that function, pain, and PGA are often considered core outcomes in RA evaluation, whereas HRQoL, fatigue, and mood disturbances are less and heterogeneously reported, besides the recognition of their importance by the Outcome Measures in Rheumatology initiative $^{25}$ or the creation of specific composite response scores by the European League Against Rheumatism. ${ }^{36}$

In order better to understand the true additional value of PROs in the management of the disease, it is important to consider their strengths and limitations. As already mentioned, PROs incorporate the patient's perspective, bringing unique 
information that cannot be collected directly by a physician. According to a recent survey, about $60 \%$ of included RA patients defined a "good day" as a day free of fatigue and/or pain, confirming the importance of these two PROs in patient perception of the disease and the potential discrepancy with rheumatologist evaluation. ${ }^{37}$ Moreover, PROs have good psychometric properties $^{38}$ and are as reproducible as joint counts $^{38,39}$ and as sensitive to change as objective disease scores. ${ }^{40}$ However, some crucial cons should be considered. Firstly, as reported by a recent systematic literature review, knowledge about the real impact of PROs as a driver for RA-treatment strategy is still limited, because of an overall lack of data. ${ }^{34}$ Moreover, other limitations may be related to the inefficiency of collecting overlapping PROs, ${ }^{41}$ challenges of tools validation, ${ }^{42}$ and unknown associations with longterm outcomes. ${ }^{43}$ Finally, PROs may be deeply influenced by confounding factors, such as concomitant fibromyalgia and/ or mood disorders, which may massively change the patient's perception of RA-related symptoms. ${ }^{44}$

\section{IL6 signaling and patient-reported outcomes}

IL6 is considered one of the most important cytokines involved in RA pathogenesis, being implicated in joint inflammation $^{45}$ and extra-articular manifestations, such as anemia, ${ }^{46}$ fatigue, ${ }^{47}$ increased insulin resistance ${ }^{48}$ and cardiovascular risk, ${ }^{49}$ and osteoporosis. ${ }^{50}$ IL6 is produced by all stromal and immune-system cells, ${ }^{16}$ and its action is explicated by the interaction with a specific receptor (IL6R $\alpha$ ), composed of a nonsignaling $\alpha$-receptor subunit existing as both membrane-bound (present only on specific cell types, such as hepatocytes, T cells, activated B cells, macrophages, and neutrophils) and soluble IL ( $\operatorname{sL6} 6 \alpha)$ and two signal-transducing Gp130 subunits that dimerize and transduce the signal through the JAK-STAT transcription pathway. ${ }^{51}$ IL6 may interact with the membrane-bound $\alpha$-subunit in the classical (cis) signaling pathway, which activates acute-phase response and is involved in infection defense, metabolic effects, and tissue regeneration. On the other hand, the interaction between the complex IL6-sIL6R and Gp130 subunits activates trans-signaling pathways on different cell types lacking the membrane-bound form (such as endothelial, smooth-muscle, and neural cells), resulting in the well-known IL6 proinflammatory effects. ${ }^{51-54}$ Therefore, sIL6R acts as an agonist, while circulating soluble Gp130 acts as an antagonist, binding IL6-sIL6R complexes and preventing trans-signaling, ${ }^{55}$ as demonstrated by the attenuation of inflammation in murine arthritis models administered with soluble Gp130. ${ }^{56}$

Neuronal cells expresses Gp130 subunits and may be stimulated by IL6 trans-signaling, so IL6 can interfere with several nervous functions, such as synaptic plasticity, neuronal development, neuronal survival, and neurogenesis. ${ }^{53}$ Moreover, IL6 has been demonstrated to exert control on the hypothalamic-pituitary-adrenal (HPA) axis. ${ }^{57}$ Those described effects on central nervous and endocrine systems suggest a direct role of IL6 in generating/amplifying mood disorders and RA symptoms, such as pain and fatigue, potentially affecting the measure of PROs. ${ }^{53,58}$

\section{IL6 and pain}

As already mentioned, pain is the hallmark of RA, represents the main reason for seeking care from a rheumatologist, and is the primary symptom for which patients try to obtain relief and meaningful improvement through therapy. ${ }^{59,60}$ Typically, arthritic pain shows peculiar features. RA patients may experience ongoing pain in the absence of any intentional stimulation or as a consequence of mechanical or thermal stimuli. The basis for this hyperalgesia lies in both the inflammatory process itself and the hypersensitization of the nociceptive system, in which the threshold for stimulation of nociceptive neurons (and thus elicitation of pain) is lowered and responses to painful stimuli heightened. ${ }^{61}$ In this scenario, proinflammatory cytokines may be involved in pain generation by both inducing/maintaining joint inflammation and targeting the nociceptive system itself, as demonstrated by the reduction in pain produced by cytokine inhibition, which is often too rapid to be attributed to the attenuation of inflammatory processes only. ${ }^{61} \mathrm{~A}$ consistent body of evidence on preclinical studies has assessed the role of IL6 as a crucial central and peripheral mediator of pain. IL6-deficient mice have been shown to exhibit less hyperalgesia in response to thermal or mechanical stimuli than wild-type mice, ${ }^{62}$ and the upregulation of spinal IL6 in models of neuropathic pain ${ }^{63}$ and inflammation ${ }^{64}$ suggests a role of spinal IL6 in inducing central pain sensitization through IL6-sIL6R trans-signaling stimulation of dorsal root ganglia, glial cells, and sensitive neurons, which all express Gp130 subunits. ${ }^{65}$ Moreover, in animal models, the injection of IL6 or IL6/sIL6R into normal joints causes a long-lasting sensitization of nociceptive C-fibers for mechanical stimuli applied to the joint. ${ }^{66,67}$ Finally, the spinal administration of soluble Gp130 attenuates the generation of spinal hyperexcitability ${ }^{68}$ and relieves pain-related behavior, ${ }^{69}$ whereas knockout mice for Gp130 
expression in sensory dorsal-root-ganglia neurons (SNSGp $130^{-/-}$) show reduced inflammation-induced pain. ${ }^{70}$ Based on all these data, IL6 plays a significant role in generation and chronicity processes of arthritic joint pain.

\section{IL6 and fatigue}

Based on recent reports, fatigue may affect up to $80 \%$ of RA patients and is a severe PRO in up to $40 \%$ of subjects. ${ }^{71,72}$ Fatigue in RA subjects may negatively influence QoL, ${ }^{73}$ and has a multidimensional origin involving inflammation, pain, anemia, poor sleep, and psychosocial factors. ${ }^{74}$ From a pathogenic point of view, fatigue may be the result of the complex interactions among different variables: disease processes, cognitive and behavioral status of patients, and personal issues in the patient's life. ${ }^{74}$ A recent literature review identified 25 possible predictors of fatigue, including cortisol response, inflammation, joint damage, muscle effort, and anemia, but highlighted that the three major variables associated with RA fatigue were pain, mood disorder (ie, depression or depressive mood), and disability. ${ }^{75}$ In this scenario, there is a growing body of evidence about the crucial role of the HPA axis and its link with proinflammatory cytokines in contributing to generate fatigue in RA patients. HPA is a main component of the stress system, responsible for the balance with physical and physiological response to stressful situations. ${ }^{76}$ HPA dysfunction-related low circulating levels of cortisol have been associated with development of fatigue symptoms. ${ }^{77}$ In normal HPA functioning, perceived stress stimulates the hypothalamic release of corticotropinreleasing hormone, which induces the adrenocorticotropic hormone secretion into the circulatory system by the anterior pituitary gland. ${ }^{78}$ Inflammatory cytokines, such as TNF, IL1, and IL6, can stimulate the HPA axis alone or synergistically during chronic inflammatory stress, such as in RA, ${ }^{79}$ with a predominant role for IL6, which is able to activate the HPA axis in humans more effectively than corticotropin-releasing hormone. ${ }^{57}$ As a consequence of this immunostimulation, untreated RA patients with high IL6 levels may show hypersecretion of adrenocorticotropic hormone without a reciprocal increase in cortisol, resulting in the development of fatigue. ${ }^{80}$ Moreover, IL6 has been demonstrated to be involved in sleep regulation, ${ }^{81}$ potentially exacerbating $\mathrm{RA}$-related fatigue. In healthy individuals, sleep deprivation produces daytime oversecretion of IL6, and administration of IL6 significantly changes sleep structure because of hypercortisolemia in the early hours of sleep. ${ }^{82}$ Finally, IL6 may contribute to fatigue in RA patients by inducing disease-associated anemia through a hepcidin-related mechanism affecting ferroportin-mediated transfer of cellular iron. ${ }^{83}$

\section{IL6, acute stress, and mood disturbances}

The importance of behavioral and mood disorders in the holistic management of RA is progressively increasing. As reported by the cross-sectional COMORA study, anxiety and depression were the most frequently observed comorbidities in a multinational cohort of 3,920 RA patients. ${ }^{5}$ Similarly, annual incidence rates of depression, anxiety disorder, and bipolar disorder were found to be significantly higher in RA subjects than in the general population, with higher frequency in females. ${ }^{84}$

The effect of acute and chronic stress on IL6 secretion has been widely studied. IL6 plasma levels are remarkably increased after acute physiological stress, such as physical exercise, ${ }^{85}$ in individuals exposed to early-life adversity ${ }^{86}$ or affected by depression. ${ }^{87}$ Data from a meta-analysis conducted on 18 studies confirmed this stress-induced increase of plasma IL6, differently to other inflammatory cytokines, such as TNF $\alpha .{ }^{88}$ Interestingly, besides immune cells, muscle, adipose tissue, and endothelial cells have been found to be possible IL6 sources in stress-related conditions. ${ }^{81}$ Considering the link with acute stress, there is convincing evidence that IL6 may be strictly related to the onset and worsening of mood disorders. Firstly, animal models lacking or knocked out for IL6 signaling or treated with IL6 blockers are resilient to social stress. ${ }^{89}$ Secondly, in healthy individuals undergoing psychological stress, low peripheral levels of IL6 predict an earlier resolution of negative mood. ${ }^{90}$ Furthermore, a recent meta-analysis confirmed IL6 as the most consistently elevated cytokine in the blood of patients with major depressive disorders, ${ }^{88}$ demonstrating a relationship between IL6mediated inflammation and depression. ${ }^{91}$ Finally, IL6 levels were elevated in cerebrospinal fluid in several conditions, as in older women with depression,,$^{92}$ patients with either depression and schizophrenia, ${ }^{93}$ suicide attempters, ${ }^{94}$ and in postpartum depression..$^{95}$ The underlying mechanism involved in IL6 contribution to depression or mood disturbances is still lacking. A possible explanation lies once again in the connection between IL6 and the HPA axis. Panic, anxiety, and depression have been associated with dysregulation of the HPA axis, ${ }^{78,96}$ and chronic stress may activate IL6 and induce a sustained corticosterone response in the hypothalamus. ${ }^{96}$ In addition, data have suggested that single-nucleotide polymorphisms in genes encoding for the IL6 promoter or IL6R may be related to an individual's increased stress sensitivity. ${ }^{97}$ 


\section{Sarilumab and PROs: data from RCTs}

The development program for sarilumab included three main RCTs conducted in different RA subpopulations. The MOBILITY trial (NCT01061736) randomized (1:1:1) 1,197 patients with inadequate response to methotrexate (Mtx) to receive sarilumab (doses of $150 \mathrm{mg}$ or $200 \mathrm{mg}$ ) or placebo every 2 weeks in combination with weekly Mtx for 52 weeks, with 24-week American College of Rheumatology $20 \%\left(\mathrm{ACR}_{20}\right)$ response, 16-week change from baseline in the HAQ-DI, and 52-week change from baseline in modified Sharp-van der Heijde score as coprimary end points. ${ }^{20-23}$ In the TARGET trial (NCT01709578), 546 patients who had experienced an inadequate response or intolerance to antiTNF therapy were randomly allocated to receive sarilumab (150 mg or $200 \mathrm{mg}$ ) or placebo every 2 weeks for 24 weeks with background conventional synthetic disease-modifying antirheumatic drugs (csDMARDs), with 24-week $\mathrm{ACR}_{20}$ and 12-week change from baseline in HAQ-DI score as coprimary end points. ${ }^{20-23}$ MONARCH (NCT02332590) is a randomized, double-blind Phase III superiority trial designed to compare sarilumab (200 mg every 2 weeks) with adalimumab (40 mg every 2 weeks) monotherapy head to head in 369 RA patients who cannot continue treatment with Mtx due to intolerance or inadequate response. The primary end point is change from baseline in $\mathrm{DAS}_{28}$ using erythrocyte-sedimentation rate at week $24 .^{20-23}$ As expected, in consideration of the previously described role of IL6 in patient perception of the disease, the three RCTs included the evaluation of several PROs as coprimary or secondary end points, most of them analyzed in post hoc analyses of the studies.

\section{MOBILITY trial and PROs}

The MOBILITY study was designed by including the evaluation of physical function assessed by the HAQ-DI in the coprimary endpoints. Compared with patients receiving placebo, patients treated with either of the sarilumab doses showed statistically significant improvements at week 16 $(P<0.0001)$ in mean change from baseline HAQ-DI scores, which were maintained through week 52. Percentages of HAQ-DI responders (defined as change from baseline in the HAQ-DI of $\geq 0.3$ ) at weeks 16,24 , and 52 were greater with both sarilumab regimens compared with placebo $(P=0.0012$ and $P<0.0001$ for sarilumab 150 and $200 \mathrm{mg}$ at week 16, respectively; $P<0.0001$ for both doses at weeks 24 and 52). ${ }^{20-23}$

Moreover, a post hoc analysis from the MOBILITY trial ${ }^{98}$ collected data on PGA, pain VAS, HAQ-DI, Functional
Assessment of Chronic Illness Therapy - fatigue (FACITF), ${ }^{99}$ and SF36 version $2,{ }^{100}$ in order to analyze HRQoL. Changes from baseline at 24 and 52 weeks were evaluated using a mixed model for repeated measures. Further post hoc analyses included proportion of patients achieving improvements equal to or greater than minimal clinically important differences (MCIDs) and normative values in the FACIT-F and SF36.

In analysis of change from baseline, patients treated with sarilumab 150 or $200 \mathrm{mg}$ reported a significant improvement in PGA, pain, and HAQ-DI scores compared with placebo since the second week of treatment, with subsequent sustained effects at both 24 and 52 weeks $(P<0.0001$; Table 1$)$. Similarly, significant improvements compared with placebo were reported in FACIT-F scores at 24 weeks with both sarilumab regimens, which were maintained till 52 weeks ( $P<0.0001$ for both doses at both time points). Sarilumab was also associated with higher $(P<0.05) 24$-week mean change from baseline in SF36 physical component summary (PCS) and mental component summary (MCS) scores compared with placebo. Greater improvements were also reported with sarilumab in all eight SF36 domains at both week 24 and week $52(P<0.05)$, with the exception of role - emotional (RE) and MCS scores with sarilumab $150 \mathrm{mg}$ at week 52 (Table 1).

In the responder analysis, the proportion of patients reporting improvement greater than or equal to MCID was higher with both sarilumab regimens vs placebo for all PROs $(P<0.05)$. Similarly, the percentage of observed improvement greater than or equal to MCID in individual SF36 domains was significantly higher with both doses of sarilumab compared with placebo across all domains $(P<0.05)$. In the vast majority (59.4\%-89.8\%) of $\mathrm{ACR}_{20}$ responders, clinically meaningful improvements in PROs were reported. The proportion of patients reporting scores greater than or equal to normative values in the FACIT-F and SF36 domains at 24 weeks was greater with sarilumab treatment in the individual domains of bodily pain, general health $(\mathrm{GH})$, social functioning, and mental health with $150 \mathrm{mg}$ and across all domains with $200 \mathrm{mg}(P<0.05)$, with the only exception being physical functioning.

\section{TARGET trial and PROs}

As previously described for the MOBILITY trial, the TARGET study included a PRO as HAQ-DI in the coprimary end points. Statistically significant improvements in mean change from baseline in HAQ-DI score at week 12 were observed in patients treated with sarilumab compared with those receiving placebo $(P=0.0007$ and $P=0.0004$ for sarilumab $150 \mathrm{mg}$ 
Table I Results of patient reported outcomes in the three randomized clinical trials of the sarilumab development program

\begin{tabular}{|c|c|c|c|c|c|c|c|c|}
\hline & $\mathbf{n}$ & Therapy & Baseline & Week & $P$-value & Week & $P$-value & Reference \\
\hline Mobility & & & & 24 & & 52 & & \\
\hline \multirow[t]{3}{*}{ Pain } & 400 & $150 \mathrm{mg} q 2 w+M t x q w$ & $65.4(21.4)$ & $-28.5(1.4)$ & $\leq 0.000 \mathrm{I}$ & $-32.7(1.4)$ & $\leq 0.000 \mathrm{I}$ & 98 \\
\hline & 399 & $200 \mathrm{mg} q 2 q+$ Mtx qw & $66.7(21.4)$ & $-31.8(1.3)$ & $\leq 0.000 \mathrm{I}$ & $-33.1(1.4)$ & $\leq 0.000 \mathrm{I}$ & \\
\hline & 398 & Placebo $q 2 w+M t x q w$ & $63.7(19.9)$ & $-15.4(1.4)$ & NS & $-19.3(1.6)$ & NS & \\
\hline \multirow[t]{3}{*}{ Fatigue } & 400 & $150 \mathrm{mg} q 2 w+M t x q w$ & $26.3(9.8)$ & $8.6(0.5)$ & $\leq 0.000 \mathrm{I}$ & $9.1(0.5)$ & $\leq 0.000 \mathrm{I}$ & 98 \\
\hline & 399 & $200 \mathrm{mg} q 2 q+M t x q w$ & $25.9(10.4)$ & $9.2(0.5)$ & $\leq 0.000 \mathrm{I}$ & $9.2(0.5)$ & $\leq 0.000 \mathrm{I}$ & \\
\hline & 398 & Placebo $q 2 w+M t x q w$ & $27.2(10.4)$ & $5.8(0.5)$ & NS & $6.1(0.5)$ & NS & \\
\hline \multirow[t]{3}{*}{ Mood disorder } & 400 & $150 \mathrm{mg} q 2 w+M t x q w$ & $39.0(11.3)$ & $5.7(0.6)$ & $<0.05$ & $7.1(0.6)$ & NS & 98 \\
\hline & 399 & $200 \mathrm{mg} q 2 w+M t x q w$ & $38.7(12.0)$ & $8.2(0.6)$ & $\leq 0.000 \mathrm{I}$ & $8.4(0.6)$ & $\leq 0.001$ & \\
\hline & 398 & Placebo $q 2 w+M t x q w$ & $38.9(11.4)$ & $3.9(0.6)$ & NS & $5.5(0.7)$ & NS & \\
\hline Target & & & & 12 & & 24 & & \\
\hline \multirow[t]{3}{*}{ Pain } & 181 & 150 mg q2w + csDMARDs & $71.0(19.3)$ & $-26.9(1.9)$ & $\leq 0.0001$ & $-31.9(2.1)$ & $\leq 0.001$ & 22 \\
\hline & 184 & $200 \mathrm{mg} q 2 \mathrm{w}+\mathrm{csDM}$ ARDs & $74.9(18.4)$ & $-30.6(1.9)$ & $\leq 0.000 \mathrm{I}$ & $-33.7(2.0)$ & $\leq 0.000 \mathrm{I}$ & \\
\hline & 181 & Placebo $q 2 w+$ csDMARDs & $71.6(18.2)$ & $-15.1(1.9)$ & NS & $-21.3(2.3)$ & NS & \\
\hline \multirow[t]{3}{*}{ Fatigue } & 181 & 150 mg q2w + csDMARDs & $23.5(10.6)$ & $8.0(0.7)$ & $<0.05$ & $9.9(0.8)$ & $<0.05$ & 22 \\
\hline & 184 & $200 \mathrm{mg} q 2 \mathrm{w}+\mathrm{csDMARDs}$ & $23.1(10.8)$ & $9.5(0.7)$ & $\leq 0.000 \mathrm{I}$ & I0.I (0.8) & $<0.05$ & \\
\hline & 181 & Placebo $q 2 w+c s D M A R D s$ & $23.7(10.8)$ & $5.6(0.7)$ & NS & $6.8(0.9)$ & NS & \\
\hline \multirow[t]{3}{*}{ Mood disorder } & 181 & 150 mg q2w + csDMARDs & $38.6(11.4)$ & $5.1(0.8)$ & NS & $6.3(0.8)$ & NS & 22 \\
\hline & 184 & $200 \mathrm{mg} q 2 \mathrm{w}+\mathrm{csDMARDs}$ & $38.6(11.4)$ & $6.5(0.7)$ & $\leq 0.000 \mathrm{I}$ & $6.8(0.8)$ & NS & \\
\hline & 181 & Placebo $q 2 w+c s D M A R D s$ & $38.5(12.6)$ & $3.5(0.7)$ & NS & $4.7(0.9)$ & NS & \\
\hline Monarch & & & & 24 & & 52 & & \\
\hline \multirow[t]{2}{*}{ Pain } & 184 & $200 \mathrm{mg}$ q2w & $70.9(18.8)$ & $-32.2(1.8)$ & $\leq 0.001$ & \multirow{2}{*}{ NR } & \multirow{2}{*}{ NR } & 23 \\
\hline & 185 & Ada $40 \mathrm{mg} \mathrm{q} 2 \mathrm{w}$ & $70.3(19.3)$ & $-27.4(1.8)$ & NS & & & \\
\hline \multirow[t]{2}{*}{ Fatigue } & 184 & $200 \mathrm{mg} q 2 \mathrm{w}$ & $23.6(8.9)$ & $10.2(0.7)$ & NS & \multirow{2}{*}{ NR } & \multirow{2}{*}{ NR } & 23 \\
\hline & 185 & Ada $40 \mathrm{mg} \mathrm{q} 2 \mathrm{w}$ & $24.4(10.3)$ & $8.4(0.7)$ & NS & & & \\
\hline \multirow[t]{2}{*}{ Mood disorder } & 184 & $200 \mathrm{mg} q 2 \mathrm{w}$ & $36.4(10.4)$ & $7.9(0.8)$ & NS & \multirow{2}{*}{ NR } & \multirow{2}{*}{ NR } & 23 \\
\hline & 185 & Ada $40 \mathrm{mg} \mathrm{q} 2 \mathrm{w}$ & 36.9 (II.6) & $6.8(0.8)$ & NS & & & \\
\hline
\end{tabular}

Note: Values given as mean (SD); qw, every week; q2w, every 2 weeks; q4w, every 4 weeks.

Abbreviations: Mtx, methotrexate; NS, not significant; csDMARDs, conventional synthetic disease-modifying antirheumatic drugs; Ada, adalimumab; NR, not reported.

and $200 \mathrm{mg}$, respectively). In addition, compared with placebo, more patients in both the sarilumab groups (sarilumab $150 \mathrm{mg}, 47.5 \%[P=0.0137]$ and sarilumab $200 \mathrm{mg}, 56.0 \%$ $[P=0.0001]$ vs placebo, $35.4 \%)$ showed clinically meaningful improvement in the HAQ-DI (defined as $\geq 0.22$ units of improvement from baseline ${ }^{101}$ at week 24). ${ }^{20-23}$

Subsequently, a post hoc analysis focusing on PRO data from the TARGET study was also performed. ${ }^{20-23}$ PROs evaluated included pain and morning-stiffness VASs, PGA, HAQ-DI, SF36, FACIT-F, Work Productivity Survey - rheumatoid arthritis (WPS-RA), and Rheumatoid Arthritis Impact of Disease (RAID). Analysis was conducted on both mean changes from baseline at weeks 12 and 24 using a mixed model for repeated measures and as proportion of patients reporting improvements greater than or equal to the MCID and normative values. Sarilumab in combination with csDMARDs provided greater improvements from baseline than placebo in PGA and pain $(P<0.0001$ at both week 12 and 24$)$, HAQ-DI $(P<0.001$ at 12 and $P<0.05$ at 24 weeks $)$, and SF36 PCS $(P<0.001$ at week 24$)$ and $\operatorname{MCS}(P<0.05$ at 12 weeks $)$.
Greater improvements with both sarilumab regimens vs placebo were observed at week 12 in FACIT-F, morning stiffness, and RAID $(P<0.05)$, which were maintained at week 24, with the exception of SF36 MCS. Significant improvements were reported with sarilumab across all SF36 domains at weeks 12 and $24(P<0.05)$, with the exceptions of GH, RE, and mental health, with $150 \mathrm{mg}$ at weeks 12 and 24 and RE with $200 \mathrm{mg}$ at week 24. Sarilumab treatment resulted in higher overall improvement in WPS-RA vs placebo at week $24(P=0.0004$ and $P=0.0003$ for 150 and $200 \mathrm{mg}$ groups, respectively), with a significant positive effect on both presenteeism (defined as days with work productivity reduced $\geq 50 \%$ ) and absenteeism. Patients receiving sarilumab $200 \mathrm{mg}$ also reported greater decrease compared with placebo in the rate of RA interference with work productivity $(2.7$ vs $1.6 ; P<0.05)$. In the responder analysis, the proportion of patients reporting improvements greater than or equal to MCID was higher with both sarilumab doses vs placebo across all PROs $(P<0.05)$, including individual SF36 domains except GH for the 150 $\mathrm{mg}$ dose and RE for both doses. ${ }^{20-23}$ 


\section{MONARCH trial and PROs}

The MONARCH trial evaluated PROs, such as HAQ-DI, SF-36 (both PCS and MCS), and FACIT-F, ${ }^{23}$ as secondary end points at 24 weeks (Table 1). The mean change in HAQ-DI score from baseline to week 24 was significantly higher in sarilumab-treated patients compared with the adalimumab group $(-0.61 \mathrm{vs}-0.43, P=0.0037)$. The proportion of patients who demonstrated a clinically meaningful improvement of $\geq 0.22$ units and the more stringent $\geq 0.3$ units was greater in the group receiving sarilumab vs patients treated with adalimumab $(P<0.01$ for both). Similarly, sarilumab-treated patients had significantly greater improvement in SF36 PCS compared with adalimumab, and improvements were observed as early as week 12 . On the other hand, both treatment groups demonstrated similar improvement in 24-week SF36 MCS and FACIT-F scores, with a trend toward greater improvement in the sarilumab group.

Finally, Gossec et al proposed a subanalysis of MONARCH and TARGET using the RAID scale for evaluating patient-perceived impact of sarilumab on RA vs either placebo plus csDMARDs or adalimumab monotherapy. ${ }^{102}$ RAID is composed of seven single-item domains, each rated by patients on an eleven-point numeric rating scale from 0 (absence) to 10 (extreme). A total score from 0 to 10 (with lower scores indicating less impact of disease) is calculated by weighting responses for each item based on patient assessment of the relative importance of the item. ${ }^{103}$ Sarilumab was superior to placebo (in TARGET) and adalimumab (in MONARCH) at weeks 12 and 24 for RAID total score (nominal $P<0.05$ for both trials), demonstrating for sarilumab a greater reduction than placebo or adalimumab monotherapy in impact of RA on patients' lives, either in combination with csDMARDs or as monotherapy. ${ }^{102}$

\section{Conclusion}

Providing additional information beyond the usual domains commonly assessed in RCTs, PROs are undoubtedly of great and rising interest in the assessment of RA and may be a promising tool for a more holistic evaluation of RA. In fact, understanding a patient's perception of the disease is crucial for the right application of shared decision-making, suggested as an overarching principle by European League Against Rheumatism recommendations on the management of RA. ${ }^{33}$ Nevertheless, besides the proven psychometric properties, more data are needed for weighing the real predictive capacities of PROs on short- and long-term outcomes and for managing potential confounding factors and discordance between patient and physician perceptions of the disease. Pain and fatigue are reported as the most important symptoms by the majority of RA patients, and together with physical function are the most frequently evaluated PROs. The link between those PROs and depression or anxiety is well described in RA, where IL6 plays a crucial role in inducing and worsening mood disturbances by both increasing inflammation and directly affecting nociceptive neurons and the HPA axis. As previously reported for other IL6 blockers, the panel of PRO data in sarilumab-treated patients is very encouraging toward more comprehensive control of both articular and extra-articular manifestation of RA. In particular, sarilumab was highly effective in improving HAQ-DI, SF36 components, and FACIT-F in all RA subpopulations, from Mtx- to TNF-inadequate-response patients. Moreover, in the head-to-head comparison provided by the MONARCH study, the effect of sarilumab monotherapy was significantly greater than adalimumab in ameliorating the same PROs, suggesting the potential superiority of IL6 over TNF blockade in the management of patient-related disease outcomes. Additional data from observational real-life research are needed for further confirmation of the potential role of sarilumab in the holistic control of RA.

\section{Disclosure}

EGF has received lecture fees from BMS, Roche, MSD, UCB, Pfizer, and AbbVie. The authors report no other conflicts of interest in this work.

\section{References}

1. Gibofsky A. Overview of epidemiology, pathophysiology, and diagnosis of rheumatoid arthritis. Am J Manag Care. 2012;18(13 Suppl):S295-S302.

2. McInnes IB, Schett G. The pathogenesis of rheumatoid arthritis. NEngl J Med. 2011;365(23):2205-2219.

3. Smolen JS, Aletaha D, McInnes IB. Rheumatoid arthritis. Lancet. 2016;388(10055):2023-2038.

4. Singh JA, Saag KG, Bridges SL Jr, et al. 2015 American College of Rheumatology guideline for the treatment of rheumatoid arthritis. Arthritis Rheumatol. 2016;68(1):1-26.

5. Dougados M, Soubrier M, Antunez A, et al. Prevalence of comorbidities in rheumatoid arthritis and evaluation of their monitoring: results of an international, cross-sectional study (COMORA). Ann Rheum Dis. 2014;73(1):62-68

6. Sturgeon JA, Finan PH, Zautra AJ. Affective disturbance in rheumatoid arthritis: psychological and disease-related pathways. Nat Rev Rheumatol. 2016;12(9):532-542.

7. Michelsen B, Kristianslund EK, Sexton J, et al. Do depression and anxiety reduce the likelihood of remission in rheumatoid arthritis and psoriatic arthritis? Data from the prospective multicentre NORDMARD study. Ann Rheum Dis. 2017;76(11):1906-1910.

8. Boer AC, Huizinga TWJ, van der Helm-van Mil AH. Depression and anxiety associate with less remission after 1 year in rheumatoid arthritis. Ann Rheum Dis. Epub 2018 Jan 8. 
9. Matcham F, Norton S, Scott DL, Steer S, Hotopf M. Symptoms of depression and anxiety predict treatment response and long-term physical health outcomes in rheumatoid arthritis: secondary analysis of a randomized controlled trial. Rheumatology. 2016;55(2):268-278.

10. Rathbun AM, Reed GW, Harrold LR. The temporal relationship between depression and rheumatoid arthritis disease activity, treatment persistence and response: a systematic review. Rheumatology. 2013;52(10):1785-1794.

11. Choy E. Understanding the dynamics: pathways involved in the pathogenesis of rheumatoid arthritis. Rheumatology. 2012;51 Suppl 5:v3-v11.

12. Desgeorges A, Gabay C, Silacci P, et al. Concentrations and origins of soluble interleukin 6 receptor-alpha in serum and synovial fluid. J Rheumatol. 1997;24(8):1510-1516.

13. Dasgupta B, Corkill M, Kirkham B, Gibson T, Panayi G. Serial estimation of interleukin 6 as a measure of systemic disease in rheumatoid arthritis. J Rheumatol. 1992;19(1):22-25.

14. Kishimoto $T$. Interleukin- 6 : from basic science to medicine -40 years in immunology. Annu Rev Immunol. 2005;23:1-21.

15. Madhok R, Crilly A, Watson J, Capell HA. Serum interleukin 6 levels in rheumatoid arthritis: correlations with clinical and laboratory indices of disease activity. Ann Rheum Dis. 1993;52(3):232-234.

16. Hunter CA, Jones SA. Corrigendum: IL-6 as a keystone cytokine in health and disease. Nat Immunol. 2017;18(11):1271.

17. Smolen JS, Beaulieu A, Rubbert-Roth A, et al. Effect of interleukin-6 receptor inhibition with tocilizumab in patients with rheumatoid arthritis (OPTION study): a double-blind, placebo-controlled, randomised trial. Lancet. 2008;371(9617):987-997.

18. Burmester GR, Feist E, Kellner H, Braun J, Iking-Konert C, RubbertRoth A. Effectiveness and safety of the interleukin 6-receptor antagonist tocilizumab after 4 and 24 weeks in patients with active rheumatoid arthritis: the first phase IIIB real-life study (TAMARA). Ann Rheum Dis. 2011;70(5):755-759.

19. Raimondo MG, Biggioggero M, Crotti C, Becciolini A, Favalli EG. Profile of sarilumab and its potential in the treatment of rheumatoid arthritis. Drug Des Devel Ther. 2017;11:1593-1603.

20. Huizinga TW, Fleischmann RM, Jasson M, et al. Sarilumab, a fully human monoclonal antibody against IL-6R $\alpha$ in patients with rheumatoid arthritis and an inadequate response to methotrexate: efficacy and safety results from the randomised SARIL-RA-MOBILITY Part A trial. Ann Rheum Dis. 2014;73(9):1626-1634.

21. Genovese MC, Fleischmann R, Kivitz AJ, et al. Sarilumab plus methotrexate in patients with active rheumatoid arthritis and inadequate response to methotrexate: results of a phase III study. Arthritis Rheumatol. 2015;67(6):1424-1437.

22. Fleischmann R, van Adelsberg J, Lin Y, et al. Sarilumab and nonbiologic disease-modifying antirheumatic drugs in patients with active rheumatoid arthritis and inadequate response or intolerance to tumor necrosis factor inhibitors. Arthritis Rheumatol. 2017;69(2):277-290.

23. Burmester GR, Lin Y, Patel R, et al. Efficacy and safety of sarilumab monotherapy versus adalimumab monotherapy for the treatment of patients with active rheumatoid arthritis (MONARCH): a randomised, double-blind, parallel-group phase III trial. Ann Rheum Dis. 2017;76(5):840-847.

24. Pincus T, Yazici Y, Bergman MJ. RAPID3, an index to assess and monitor patients with rheumatoid arthritis, without formal joint counts: similar results to DAS28 and CDAI in clinical trials and clinical care. Rheum Dis Clin North Am. 2009;35(4):773-778.

25. Kirwan JR, Boonen A, Harrison MJ, et al. OMERACT 10 Patient Perspective Virtual Campus - Valuing Health: measuring outcomes in rheumatoid arthritis fatigue, RA sleep, arthroplasty, and systemic sclerosis; and clinical significance of changes in health. J Rheumatol. 2011;38(8):1728-1734.

26. Barton JL, Imboden J, Graf J, Glidden D, Yelin EH, Schillinger D. Patient-physician discordance in assessments of global disease severity in rheumatoid arthritis. Arthritis Care Res (Hoboken). 2010;62(6):857-864.
27. Gossec L, Dougados M, Rincheval N, et al. Elaboration of the preliminary Rheumatoid Arthritis Impact of Disease (RAID) score: a EULAR initiative. Ann Rheum Dis. 2009;68(11):1680-1685.

28. van Tuyl LH, Hewlett S, Sadlonova M, et al. The patient perspective on remission in rheumatoid arthritis: 'You've got limits, but you're back to being you again'. Ann Rheum Dis. 2015;74(6):1004-1010.

29. Bartlett SJ, Hewlett S, Bingham CO, et al. Identifying core domains to assess flare in rheumatoid arthritis: an OMERACT international patient and provider combined Delphi consensus. Ann Rheum Dis. 2012;71(11):1855-1860.

30. Balogh E, Dias J, Orr C, et al. Comparison of remission criteria in a tumour necrosis factor inhibitor treated rheumatoid arthritis longitudinal cohort: patient global health is a confounder. Arthritis Res Ther. 2013;15(6):R221.

31. Anderson J, Caplan L, Yazdany J, et al. Rheumatoid arthritis disease activity measures: American College of Rheumatology recommendations for use in clinical practice. Arthritis Care Res (Hoboken). 2012;64(5):640-647.

32. Stoffer MA, Smolen JS, Woolf A, et al. Development of patient-centred standards of care for osteoarthritis in Europe: the eumusc.net-project. Ann Rheum Dis. 2015;74(6):1145-1149.

33. Smolen JS, Landewé R, Bijlsma J, et al. EULAR recommendations for the management of rheumatoid arthritis with synthetic and biological disease-modifying antirheumatic drugs: 2016 update. Ann Rheum Dis. 2017;76(6):960-977.

34. Kilic L, Erden A, Bingham CO, Gossec L, Kalyoncu U. The reporting of patient-reported outcomes in studies of patients with rheumatoid arthritis: a systematic review of 250 articles. J Rheumatol. 2016;43(7):1300-1305.

35. Kalyoncu U, Dougados M, Daures JP, Gossec L. Reporting of patientreported outcomes in recent trials in rheumatoid arthritis: a systematic literature review. Ann Rheum Dis. 2009;68(2):183-190.

36. Gossec L, Paternotte S, Aanerud GJ, et al. Finalisation and validation of the rheumatoid arthritis impact of disease score, a patient-derived composite measure of impact of rheumatoid arthritis: a EULAR initiative. Ann Rheum Dis. 2011;70(6):935-942.

37. Strand V, Wright GC, Bergman MJ, Tambiah J, Taylor PC. Patient expectations and perceptions of goal-setting strategies for disease management in rheumatoid arthritis. J Rheumatol. 2015;42(11):2046-2054.

38. Boers M, Kirwan JR, Wells G, et al. Developing core outcome measurement sets for clinical trials: OMERACT Filter 2.0. J Clin Epidemiol. 2014;67(7):745-753.

39. Tugwell P, Wells G, Strand V, et al. Clinical improvement as reflected in measures of function and health-related quality of life following treatment with leflunomide compared with methotrexate in patients with rheumatoid arthritis: sensitivity and relative efficiency to detect a treatment effect in a twelve-month, placebo-controlled trial. Arthritis Rheum. 2000;43(3):506-514.

40. Wells G, Li T, Maxwell L, Maclean R, Tugwell P. Responsiveness of patient reported outcomes including fatigue, sleep quality, activity limitation, and quality of life following treatment with abatacept for rheumatoid arthritis. Ann Rheum Dis. 2008;67(2):260-265.

41. Gossec L, Dougados M, Dixon W. Patient-reported outcomes as end points in clinical trials in rheumatoid arthritis. RMD Open. 2015;1(1):e000019.

42. Brundage M, Blazeby J, Revicki D, et al. Patient-reported outcomes in randomized clinical trials: development of ISOQOL reporting standards. Qual Life Res. 2013;22(6):1161-1175.

43. Felson DT, Smolen JS, Wells G, et al. American College of Rheumatology/European League Against Rheumatism provisional definition of remission in rheumatoid arthritis for clinical trials. Ann Rheum Dis. 2011;70(3):404-413.

44. Coury F, Rossat A, Tebib A, et al. Rheumatoid arthritis and fibromyalgia: a frequent unrelated association complicating disease management. J Rheumatol. 2009;36(1):58-62.

45. Fonseca JE, Santos MJ, Canhão H, Choy E. Interleukin-6 as a key player in systemic inflammation and joint destruction. Autoimmun Rev. 2009;8(7):538-542. 
46. Song SN, Tomosugi N, Kawabata H, Ishikawa T, Nishikawa T, Yoshizaki K. Down-regulation of hepcidin resulting from long-term treatment with an anti-IL-6 receptor antibody (tocilizumab) improves anemia of inflammation in multicentric Castleman disease. Blood. 2010;116(18):3627-3634.

47. Gossec L, Steinberg G, Rouanet S, Combe B. Fatigue in rheumatoid arthritis: quantitative findings on the efficacy of tocilizumab and on factors associated with fatigue - the French multicentre prospective PEPS study. Clin Exp Rheumatol. 2015;33(5):664-670.

48. Fève B, Bastard JP. The role of interleukins in insulin resistance and type 2 diabetes mellitus. Nat Rev Endocrinol. 2009;5(6):305-311.

49. Danesh J, Kaptoge S, Mann AG, et al. Long-term interleukin-6 levels and subsequent risk of coronary heart disease: two new prospective studies and a systematic review. PLoS Med. 2008;5(4):e78.

50. Abdel Meguid MH, Hamad YH, Swilam RS, Barakat MS. Relation of interleukin-6 in rheumatoid arthritis patients to systemic bone loss and structural bone damage. Rheumatol Int. 2013;33(3):697-703.

51. Rose-John S. IL-6 trans-signaling via the soluble IL-6 receptor: importance for the pro-inflammatory activities of IL-6. Int J Biol Sci 2012;8(9):1237-1247.

52. Rose-John S, Winthrop K, Calabrese L. The role of IL-6 in host defence against infections: immunobiology and clinical implications. Nat Rev Rheumatol. 2017;13(7):399-409.

53. Marz P, Cheng J-G, Gadient RA, et al. Sympathetic neurons can produce and respond to interleukin 6. Proc Natl Acad Sci U S A. 1998;95(6): 3251-3256.

54. Jostock T, Müllberg J, Ozbek S, et al. Soluble gp130 is the natural inhibitor of soluble interleukin-6 receptor transsignaling responses. Eur J Biochem. 2001;268(1):160-167.

55. Jones SA, Richards PJ, Scheller J, Rose-John S. IL-6 transsignaling: the in vivo consequences. J Interferon Cytokine Res. 2005;25(5):241-253.

56. Nowell MA, Richards PJ, Horiuchi S, et al. Soluble IL-6 receptor governs IL-6 activity in experimental arthritis: blockade of arthritis severity by soluble glycoprotein 130. J Immunol. 2003;171(6):3202-3209.

57. Mastorakos G, Chrousos GP, Weber JS. Recombinant interleukin-6 activates the hypothalamic-pituitary-adrenal axis in humans. J Clin Endocrinol Metab. 1993;77(6):1690-1694.

58. März P, Otten U, Rose-John S. Neural activities of IL-6-type cytokines often depend on soluble cytokine receptors. Eur J Neurosci. 1999;11(9):2995-3004.

59. Lee YC. Effect and treatment of chronic pain in inflammatory arthritis. Curr Rheumatol Rep. 2013;15(1):300.

60. Heiberg T, Finset A, Uhlig T, Kvien TK. Seven year changes in health status and priorities for improvement of health in patients with rheumatoid arthritis. Ann Rheum Dis. 2005;64(2):191-195.

61. Schaible HG. Nociceptive neurons detect cytokines in arthritis. Arthritis Res Ther. 2014;16(5):470.

62. Xu HJ, Hao JX, Andell-Jonsson S, Poli V, Bartfai T, WiesenfeldHallin Z. Nociceptive responses in interleukin-6-deficient mice to peripheral inflammation and peripheral nerve section. Cytokine. 1997;9(12):1028-1033.

63. Wieseler-Frank J, Maier SF, Watkins LR. Central proinflammatory cytokines and pain enhancement. Neurosignals. 2005;14(4): 166-174.

64. Bao L, Zhu Y, Elhassan AM, et al. Adjuvant-induced arthritis: IL-1 $\beta$, IL-6 and TNF- $\alpha$ are up-regulated in the spinal cord. Neuroreport. 2001;12(18):3905-3908.

65. Obreja O, Biasio W, Andratsch M, et al. Fast modulation of heatactivated ionic current by proinflammatory interleukin 6 in rat sensory neurons. Brain. 2005;128(7):1634-1641.

66. Watkins LR, Wiertelak EP, Goehler LE, Smith KP, Martin D, Maier SF. Characterization of cytokine-induced hyperalgesia. Brain Res. 1994;654(1):15-26.

67. Brenn D, Richter F, Schaible HG. Sensitization of unmyelinated sensory fibers of the joint nerve to mechanical stimuli by interleukin-6 in the rat: an inflammatory mechanism of joint pain. Arthritis Rheum. 2007;56(1):351-359.
68. Vazquez E, Kahlenbach J, Banchet GS, König C, Schaible HG, Ebersberger A. Spinal interleukin-6 is an amplifier of arthritic pain in the rat. Arthritis Rheum. 2012;64(7):2233-2242.

69. Boettger MK, Leuchtweis J, Kümmel D, Gajda M, Bräuer R, Schaible HG. Differential effects of locally and systemically administered soluble glycoprotein 130 on pain and inflammation in experimental arthritis. Arthritis Res Ther. 2010;12(4):R140.

70. Andratsch M, Mair N, Constantin CE, et al. A key role for gp130 expressed on peripheral sensory nerves in pathological pain. $J \mathrm{Neu}$ rosci. 2009;29(43):13473-13483.

71. Townes SV, Furst DE, Thenkondar A. The impact of tocilizumab on physical function and quality of life in patients with rheumatoid arthritis: a systematic literature review and interpretation. Open Access Rheumatol. 2012;4:87-92.

72. Cramp F, Hewlett S, Almeida C, et al. Non-pharmacological interventions for fatigue in rheumatoid arthritis. Cochrane Database Syst Rev. 2013;8:CD008322.

73. Alikari V, Sachlas A, Giatrakou S, et al. Fatigue in arthritis: a multidimensional phenomenon with impact on quality of life: fatigue and quality of life in arthritis. Adv Exp Med Biol. 2017;987:243-256.

74. Hewlett S, Chalder T, Choy E, et al. Fatigue in rheumatoid arthritis: time for a conceptual model. Rheumatology. 2011;50(6):1004-1006.

75. Nikolaus S, Bode C, Taal E, van de Laar MA. Fatigue and factors related to fatigue in rheumatoid arthritis: a systematic review. Arthritis Care Res (Hoboken). 2013;65(7):1128-1146.

76. Silverman MN, Pearce BD, Biron CA, Miller AH. Immune modulation of the hypothalamic-pituitary-adrenal (HPA) axis during viral infection. Viral Immunol. 2005;18(1):41-78.

77. Papadopoulos AS, Cleare AJ. Hypothalamic-pituitary-adrenal axis dysfunction in chronic fatigue syndrome. Nat Rev Endocrinol. 2012;8(1):22-32

78. Du X, Pang TY. Is dysregulation of the HPA-axis a core pathophysiology mediating co-morbid depression in neurodegenerative diseases? Front Psychiatry. 2015;6:32.

79. Tsigos C, Chrousos GP. Hypothalamic-pituitary-adrenal axis, neuroendocrine factors and stress. J Psychosom Res. 2002;53(4):865-871.

80. Eijsbouts AM, van den Hoogen FH, Laan RF, Hermus AR, Sweep CG, van de Putte LB. Hypothalamic-pituitary-adrenal axis activity in patients with rheumatoid arthritis. Clin Exp Rheumatol. 2005;23(5):658-664.

81. Rohleder N, Aringer M, Boentert M. Role of interleukin-6 in stress, sleep, and fatigue. Ann N Y Acad Sci. 2012;1261(1):88-96.

82. Vgontzas AN, Papanicolaou DA, Bixler EO, et al. Circadian interleukin-6 secretion and quantity and depth of sleep. J Clin Endocrinol Metab. 1999;84(8):2603-2607.

83. Weiss G, Goodnough LT. Anemia of chronic disease. $N$ Engl J Med. 2005;352(10):1011-1023.

84. Marrie RA, Walld R, Bolton JM, et al. Physical comorbidities increase the risk of psychiatric comorbidity in immune-mediated inflammatory disease. Gen Hosp Psychiatry. 2018;51:71-78.

85. Papanicolaou DA, Petrides JS, Tsigos C, et al. Exercise stimulates interleukin-6 secretion: inhibition by glucocorticoids and correlation with catecholamines. Am J Physiol Endocrinol Metab. 1996;271(3):E601-E605

86. Carpenter LL, Gawuga CE, Tyrka AR, Lee JK, Anderson GM, Price LH. Association between plasma IL-6 response to acute stress and early-life adversity in healthy adults. Neuropsychopharmacology. 2010;35(13):2617-2623.

87. Pace TW, Mletzko TC, Alagbe O, et al. Increased stress-induced inflammatory responses in male patients with major depression and increased early life stress. Am J Psychiatry. 2006;163(9):1630-1633.

88. Steptoe A, Hamer M, Chida Y. The effects of acute psychological stress on circulating inflammatory factors in humans: a review and meta-analysis. Brain Behav Immun. 2007;21(7):901-912.

89. Chourbaji S, Urani A, Inta I, et al. IL-6 knockout mice exhibit resistance to stress-induced development of depression-like behaviors. Neurobiol Dis. 2006;23(3):587-594. 
90. Späth-Schwalbe E, Hansen K, Schmidt F, et al. Acute effects of recombinant human interleukin- 6 on endocrine and central nervous sleep functions in healthy men. J Clin Endocrinol Metab. 1998;83(5):1573-1579.

91. Khandaker GM, Pearson RM, Zammit S, Lewis G, Jones PB. Association of serum interleukin 6 and $\mathrm{C}$-reactive protein in childhood with depression and psychosis in young adult life. JAMA Psychiatry. 2014;71(10):1121-1128.

92. Kern S, Skoog I, Börjesson-Hanson A, et al. Higher CSF interleukin-6 and CSF interleukin-8 in current depression in older women: results from a population-based sample. Brain Behav Immun. 2014;41:55-58.

93. Sasayama D, Hattori K, Wakabayashi C, et al. Increased cerebrospinal fluid interleukin-6 levels in patients with schizophrenia and those with major depressive disorder. J Psychiatr Res. 2013;47(3):401-406.

94. Lindqvist D, Janelidze S, Hagell P, et al. Interleukin-6 is elevated in the cerebrospinal fluid of suicide attempters and related to symptom severity. Biol Psychiatry. 2009;66(3):287-292.

95. Boufidou F, Lambrinoudaki I, Argeitis J, et al. CSF and plasma cytokines at delivery and postpartum mood disturbances. J Affect Disord. 2009;115(1-2):287-292.

96. Girotti M, Donegan JJ, Morilak DA. Influence of hypothalamic IL-6/ gp130 receptor signaling on the HPA axis response to chronic stress. Psychoneuroendocrinology. 2013;38(7):1158-1169.

97. Hodes GE, Ménard C, Russo SJ. Integrating interleukin-6 into depression diagnosis and treatment. Neurobiol Stress. 2016;4:15-22.
98. Strand V, Kosinski M, Chen CI, et al. Sarilumab plus methotrexate improves patient-reported outcomes in patients with active rheumatoid arthritis and inadequate responses to methotrexate: results of a phase III trial. Arthritis Res Ther. 2016;18:198.

99. Cella D, Yount S, Sorensen M, Chartash E, Sengupta N, Grober J. Validation of the functional assessment of Chronic Illness Therapy Fatigue Scale relative to other instrumentation in patients with rheumatoid arthritis. J Rheumatol. 2005;32(5):811-819.

100. Ware JE, Bjorner JB, Turner-Bowker D, Maruish ME. User's Manual for the SF-36v2 Health Survey. 2nd ed. Lincoln (RI): Quality Metric Incorporated; 2007.

101. Kosinski M, Zhao SZ, Dedhiya S, Osterhaus JT, Ware JE. Determining minimally important changes in generic and disease-specific healthrelated quality of life questionnaires in clinical trials of rheumatoid arthritis. Arthritis Rheum. 2000;43(7):1478-1487.

102. Gossec LSV, Proudfoot C, Chen C, et al. Rheumatoid arthritis (RA) impact following treatment with sarilumab: patient reported outcomes using the RAID scale from two randomized phase III trials. Ann Rheum Dis. 2017;76 Suppl 2:575-576.

103. Dougados M, Brault Y, Logeart I, van der Heijde D, Gossec L, Kvien T. Defining cut-off values for disease activity states and improvement scores for patient-reported outcomes: the example of the Rheumatoid Arthritis Impact of Disease (RAID). Arthritis Res Ther. 2012;14(3):R129.
Patient Related Outcome Measures

\section{Publish your work in this journal}

Patient Related Outcome Measures is an international, peer-reviewed, open access journal focusing on treatment outcomes specifically relevant to patients. All aspects of patient care are addressed within the journal and practitioners from all disciplines are invited to submit their work as well as healthcare researchers and patient support groups.

\section{Dovepress}

The journal is included in PubMed. The manuscript management system is completely online and includes a very quick and fair peer-review system. Visit http://www.dovepress.com/testimonials.php to read real quotes from published authors. 